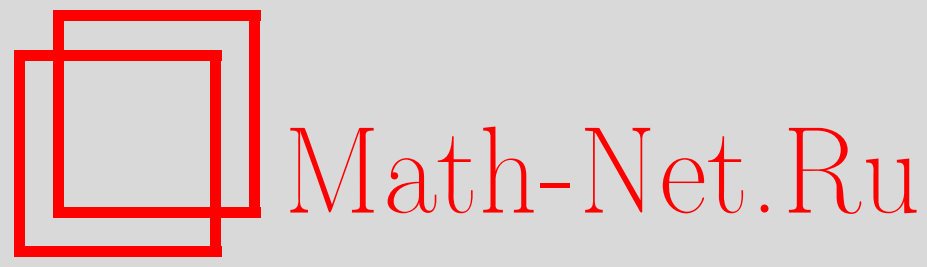

В. А. Клепцын, М. Б. Нальский, Устойчивость существования негиперболических мер для $C^{1}$ диффеоморфизмов, Функи. анализ и его прил., 2007, том 41, выпуск 4, 30-45

DOI: https://doi.org/10.4213/faa2877

Использование Общероссийского математического портала MathNet.Ru подразумевает, что вы прочитали и согласны с пользовательским соглашением

http://www . mathnet.ru/rus/agreement

Параметры загрузки:

IP : 34.229 .108 .108

26 апреля 2023 г., 18:29:36

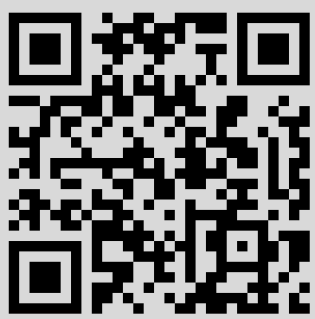




\title{
Устойчивость существования негиперболических мер для $C^{1}$-диффеоморфизмов*
}

\author{
(c) 2007. В. А. КлЕПцЫН, М. Б. НАЛЬСКИЙ
}

\section{§1. Введение}

Гиперболические динамические системы по определению имеют ненулевые показатели Ляпунова. Они, однако, не являются типичными в пространстве всех динамических систем [1]. Более широкий класс - неравномерно гиперболические системы, изучаемые в теории Песина [2]. Они также имеют ненулевые показатели Ляпунова и также нетипичны.

Более подробно, теория Песина изучает диффеоморфизмы, имеющие ненулевые показатели Ляпунова относительно некоторой фиксированной инвариантной меры, и описывает поведение траекторий, типичных относительно этой меры. Инвариантная мера может быть задана изначально и согласована с гладкой структурой, а может определяться динамической системой. Эти два случая существенно отличаются друг от друга.

Для $C^{1}$-диффеоморфизмов двумерных многообразий, сохраняющих площадь, Боки [7] обнаружил, что типичные отображения этого класса либо являются аносовскими, либо имеют нулевые показатели Ляпунова.

Удивительное свойство сохраняющих объем диффеоморфизмов в размерности выше двух было открыто Шубом и Уилкинсон [10]. Они нашли открытое множество частично гиперболических отображений трехмерного тора в себя со следующим свойством. Отображения из этого множества имеют инвариантное слоение, слои которого - окружности. Эти слои непрерывно, но не абсолютно непрерывно зависят от начального условия. Между тем почти все (в смысле инвариантной меры Лебега) орбиты каждого из этих отображений имеют ненулевые показатели Ляпунова (два положительных, один отрицательный). Парадоксальный факт заключается в том, что множество полной меры, состоящее из этих орбит, пересекает каждый слой по множеству меры 0 на окружности. Позже А. Каток обнаружил, а Рюэлль и Уилкинсон [11] доказали, что это пересечение состоит из конечного числа точек.

Для частично-гиперболических систем, сохраняющих объем, при некоторых дополнительных предположениях Баравьера и Бонатти [9] доказали, что $C^{1}$-малым возмущением в классе таких систем можно устранить нулевой центральный показатель Ляпунова для сохраняемого объема как инвариантной меры. Перечисленные выше результаты относятся к так называемой консервативной динамике.

*Работа осуществлена при частичной поддержке грантов CRDF RM1-2358-MO-02, РФФИ 07-01-00017-а и НЦНИЛ_а 05-01-02801; первый автор также получил поддержку Swiss National Science Foundation. 
Наша работа относится к «неконсервативному подходу» - исследованию систем, инвариантные меры которых определяются динамикой и могут быть не согласованы с гладкой структурой. Одна из важных проблем неконсервативной динамики была поставлена в [10]: «Верно ли, что типичный диффеоморфизм имеет ненулевые показатели Ляпунова относительно всех «хороших» инвариантных мер?» «Хорошими» называются инвариантные меры, получаемые как частичные пределы временны́х средних меры Лебега (процедура КрыловаБоголюбова).

До последнего времени даже более простой вопрос, верно ли, что типичный дифбеоморфизм имеет ненулевые показатели Ляпунова относительно всех инвариантных мер, оставался открытым. Мы даем отрицательный ответ на этот вопрос. Наша статья продолжает работу [18].

Ранее Городецкий и Ильяшенко [14]-[17] доказали, что наличие одной орбиты с нулевым центральным показателем Ляпунова может быть неустранимо малым шевелением.

\section{§2. Основные результаты}

В настоящей статье доказана, в определенном смысле (см. теорему 1 ниже), неустранимость нулевых показателей Ляпунова. Мы следуем стратегии, предложенной Городецким и Ильяшенко. Сначала новое явление обнаруживается для ступенчатых косых произведений (определения напоминаются в §3). Затем использованная техника адаптируется к случаю мягких косых произведений. Наконец, с помощью техники гладкой реализации отсюда выводится соответствующий результат для гладких систем. Первый шаг - исследование показателей Ляпунова для ступенчатых косых произведений - сделан в [18]. В этой статье сделаны второй и третий шаги:

Теорема 1 (основной результат). Для замкнутого многообразия $M, \operatorname{dim} M$ $\geqslant 3$, существует область $U \subset \operatorname{Diff}^{1}(M)$, любой дифбеоморбизм $f$ из которой обладает следующим свойством. Найдутся такие локально максимальное частично гиперболическое инвариантное множество $\Lambda \subset M$ иеатомарная эргодическая инвариантная мера $\mu, \operatorname{supp} \mu \subset \Lambda$, что один из показателей Ляпунова дифбеоморбизма $f$ относительно меры $\mu$ равен нулю.

2.1. Схема доказательства основного результата. В этом разделе мы приводим набросок доказательства основного результата. Эта теорема, как уже было сказано выше, доказывается как объединение следующих двух теорем. Первая из них (строго сформулированная в §4) устанавливает типичность нулевых показателей Ляпунова для мягких косых произведений:

Теорема 2. Существует открытая область в пространстве гёльдеровых мягких косъх произведений над подковой Смейла, включающая в себя ступенчатые косые произведения, сколь угодно близкие к тождественным, любое произведение из которой имеет меру с нулевым показателем Ляпунова вдоль слоя.

Это утверждение доказывается методами, аналогичными примененным в работе [18] для ступенчатых косых произведений. А именно, с помощью некоторой итеративной процедуры строится последовательность периодических орбит с возрастающими периодами. В этой последовательности следующие орбиты все 
более «похожи» на предыдущие (точнее, на пройденные несколько раз подряд предыдущие). При этом показатели Ляпунова, соответствующие этим орбитам, стремятся к нулю. В результате последовательность мер, соответствующих этим орбитам, слабо сходится к некоторой эргодической мере, показатель Ляпунова которой равен нулю.

Вторая из упомянутых выше теорем - результат, принадлежащий А. С. Городецкому [16], - сформулирована строго в $§ 5$. Грубо говоря, она утверждает, что при определенных условиях наличие у гладкой динамической системы (локально максимального частично гиперболического) инвариантного множества, динамика на котором есть мягкое косое произведение, сохраняется при малых возмущениях, а мягкие косые произведения для возмущенных систем гёльдеровы и близки к исходному.

Основной результат выводится из этих двух теорем следующим образом: рассматривается гладкая реализация подковы Смейла, над которой строится ступенчатое гладкое косое произведение из области, обеспеченной теоремой 2. Для каждого диффеоморфизма, достаточно близкого к построенному, найдется подмножество, динамика на котором сопряжена гёльдерову мягкому косому произведению, близкому к исходному. Это косое произведение, таким образом, принадлежит той же области. Поэтому для него найдется инвариантная мера с нулевым показателем Ляпунова вдоль слоя. Отсюда следует, что для соответствующей инвариантной меры возмущенного диффеоморфизма один из показателей Ляпунова равен нулю.

Используемая нами в этих рассуждениях техника гладких реализаций была разработана А. С. Городецким и Ю. С. Ильяшенко в [14]-[16].

2.2. Благодарности. Авторы глубоко признательны Ю. С. Ильяшенко за терпение и всестороннюю поддержку настоящей работы и А. С. Городецкому за полезные идеи и обсуждения.

2.3. План статьи. В $\S 3$ мы вводим обозначения и определения. В $\S 4$ мы строго формулируем теорему 2 и описываем идею и ход ее доказательства. В $\S 5$, используя технику гладкой реализации и теорию частично гиперболических систем, мы выводим из теоремы 2 теорему 1. Наконец, в $\S 6-9$ мы доказываем теорему 2 .

\section{§3. Обозначения и определения}

Пусть $\Sigma^{N}-$ пространство двусторонних последовательностей из символов $\{0, \ldots, N-1\}$, а $\sigma-$ сдвиг Бернулли $\Sigma^{N} \rightarrow \Sigma^{N}$. Мы снабдим $\Sigma^{N}$ метрикой $d_{\Sigma^{N}}\left(\omega, \omega^{\prime}\right)=2^{-\min \left\{|n|: \omega_{n} \neq \omega_{n}^{\prime}\right\}}$. Положим $M=\Sigma^{N} \times S^{1}$.

Пусть $g_{i}(i=0, \ldots, N-1)$ - диффеоморфизмы $S^{1} \rightarrow S^{1}$. Рассмотрим отображение

$$
G: M \rightarrow M, \quad(\omega, x) \rightarrow\left(\sigma \omega, g_{\omega_{0}}(x)\right) .
$$

Заметим, что отображение $G$, применяемое к слою над точкой $\omega$ в базе, зависит не от всей последовательности $\omega$, а только от ее элемента $\omega_{0}$. Будем называть такие косые произведения ступенчатыми косыми произведениями.

Пусть $f_{\omega}: S^{1} \rightarrow S^{1}, \omega \in \Sigma^{N}$, - семейство диффеоморфизмов окружности. Рассмотрим также отображение

$$
F: M \rightarrow M, \quad(\omega, x) \rightarrow\left(\sigma \omega, f_{\omega}(x)\right) .
$$


Мы называем такие косые произведения мягкими, поскольку отображение в слое зависит от всего слова $\omega$ и не определяется никакой его конечной частью.

Определение 1. Мягкое косое произведение $F$, заданное формулой $(2)$, называется $(C, \alpha)$-гёлъдеровъм, если

$$
\forall \omega, \omega^{\prime} \in \Sigma^{N} \quad d_{C^{0}}\left(f_{\omega}, f_{\omega^{\prime}}\right)<C \cdot d_{\Sigma^{N}}\left(\omega, \omega^{\prime}\right)^{\alpha} .
$$

Определение 2. ( $L, C, \alpha)$-системa - это $(C, \alpha)$-гёльдерово косое произведение, отображения $f_{\omega}$ которого непрерывно в $\operatorname{Diff}^{1}$-норме зависят от $\omega$ (в частности, гладкое вдоль слоев), такое, что имеет место следующая оценка на максимальную скорость растяжения:

$$
\forall \omega \in \Sigma^{N} \quad \max _{x \in S^{1}} \max \left(f_{\omega}^{\prime}(x),\left(f_{\omega}^{-1}\right)^{\prime}(x)\right)<L .
$$

Мы будем рассматривать на пространстве $(L, C, \alpha)$-систем метрику равномерного Diff ${ }^{1}$-расстояния

$$
d(F, \tilde{F})=\sup _{\omega \in \Sigma^{n}} d_{\operatorname{Diff}^{1}}\left(f_{\omega}, \tilde{f}_{\omega}\right) .
$$

Введем обозначения

$$
\bar{f}_{m}[\omega]=f_{\sigma^{m-1} \omega} \circ \cdots \circ f_{\sigma \omega} \circ f_{\omega}, \quad \bar{f}_{-m}[\omega]=f_{\sigma^{-m} \omega}^{-1} \circ \cdots \circ f_{\sigma^{-1} \omega}^{-1}, \quad \bar{f}_{0}[\omega]=\mathrm{id} .
$$

Определение 3. Пусть $F-$ косое произведение вида (2). Показателем Ляпунова вдоль слоя в точке $(\omega, x)$ называется следующая функция (определенная в тех точках, где предел существует):

$$
\lambda^{c}(\omega, x):=\lim _{n \rightarrow \infty} \frac{1}{n} \ln \left|D \bar{f}_{n}[\omega](x)\right| .
$$

Если отображение $F$ обладает эргодической вероятностной мерой $\nu$, то найдется множество $\nu$-полной меры, такое, что для всех точек из этого множества показатель Ляпунова вдоль слоя определен и не зависит от точки множества. В этом случае функция $\lambda^{c}(\omega, x)$ - константа на множестве $\nu$-полной меры и можно говорить о показателе Ляпунова вдоль слоя относительно меры $\nu$ (обозначение $\left.\lambda^{c}(\nu)\right)$.

\section{4. Основной результат для мягких косых произведений}

Теорема 2. При числе символов $N \geqslant 5$ для любых $L>1, C>0,0<\alpha<1$ в пространстве $(L, C, \alpha)$-систем найдется открытая область, кажсдая система из которой обладает неатомарной эргодической инвариантной мерой с нулевым показателем Ляпунова вдоль слоя. Эта область содержит ступенчатые косые произведения, сколь угодно близкие к тождественному (вдоль слоя).

Для доказательства теоремы 2 мы будем строить искомую меру как предел мер, равномерно распределенных на периодических орбитах, показатели Ляпунова которых стремятся к нулю. Кроме того, периодические орбиты будут в некотором смысле «похожи» друг на друга.

Идея реализуется следующим образом. В $\$ 6$ мы, следуя А. С. Городецкому и Ю. С. Ильяшенко (см. [15], [16]), исследуем расхождение орбит мягких косых произведений. В $§ 7$ описывается конструкция, позволяющая по одной периодической орбите построить другую, с бо́льшим периодом, меньшим (по модулю) 
показателем Ляпунова и достаточно «похожую» на исходную. В 88 по индукции строится требуемая последовательность орбит, обосновывается эргодичность и неатомарность предельной меры и показывается (как следствие эргодичности), что показатель Ляпунова вдоль слоя для предельной меры есть предел показателей Ляпунова. Наконец, в $\$ 9$ мы строим диффеоморфизмы, удовлетворяющие условиям $\S 6$.

\section{§5. Гладкая реализация и доказательство теоремы 1}

Рассмотрим подкову Смейла, реализованную как отображение попарно не пересекающихся прямоугольников. А именно, пусть $B=[0,1] \times[0,1]-$ единичный прямоугольник на координатной плоскости. Разделим его на 11 равных вертикальных прямоугольников $C_{k}, k=0, \ldots, 10$, и положим $D_{i}:=C_{2 i+1}$. Разделим тот же прямоугольник на 11 равных горизонтальных прямоугольников $C_{k}^{\prime}$ и положим $D_{i}^{\prime}:=C_{2 i+1}^{\prime}$.

Пусть $D=\bigcup_{i=0}^{4} D_{i}, D^{\prime}=\bigcup_{i=0}^{4} D_{i}^{\prime}$. Пусть отображение $T: D \rightarrow D^{\prime}$ действует на каждом из прямоугольников $D_{i}$ линейным сжатием по вертикали и линейным растяжением по горизонтали, переводя его в соответствующий прямоугольник $D_{i}^{\prime}$.

Хорошо известно, что отображение $T$ имеет инвариантное множество $\Lambda$, гомеоморфное $\Sigma^{5}$, и $\left.T\right|_{\Lambda}$ сопряжено сдвигу Бернулли $\sigma: \Sigma^{5} \rightarrow \Sigma^{5}$. Мы обозначим сопрягающий гомеоморфизм через $\Phi_{0}: \Lambda \rightarrow \Sigma^{5}$.

По заданным диффеоморфизмам $\left\{g_{i}\right\}_{i=0, \ldots, 4}$ окружности мы можем построить гладкую реализацию ступенчатого косого произведения (1). А именно, положим

$$
\mathscr{F}: D \times S^{1} \rightarrow D^{\prime} \times S^{1}, \quad \mathscr{F}(z, x)=\left(T(z), g_{j}(x)\right) \text { при } z \in D_{j} .
$$

Легко видеть, что $\left.\mathscr{F}\right|_{\Lambda \times S^{1}}$ сопряжено отображению (1). Множество $\Lambda \times S^{1}$ является (при достаточно близких к тождественному отображениях $g_{i}$ ) частично гиперболическим для $\mathscr{F}$, а его центральные слои являются слоями проекции на первый сомножитель: $\Lambda \times S^{1} \rightarrow \Lambda, \Lambda \simeq \Sigma^{5}$.

Теорема 3 (Городецкий [16]). Пусть $C^{r+1}$-гладкое $(0 \leqslant r \leqslant \infty)$ отображсение $T: D \rightarrow D^{\prime}$ является гиперболическим с локально максимальным множеством $\Lambda, \Lambda=\bigcap_{n \in \mathbb{Z}} T^{n}(D)$, а $M$ - замкнутое многообразие. Рассмотрим отображение $\mathscr{F}_{0}: D \times M \rightarrow D^{\prime} \times M, \mathscr{F}_{0}=T \times \mathrm{id}_{M}$. Тогда существует $C^{r+1}$ окрестность $V$ диффеоморфизма $\mathscr{F}_{0}$, такая, что для любого диффеоморфизма $\mathscr{B} \in V$

1) существует инвариантное подмножество $\Delta_{\mathscr{B}}$, гомеоморфное $\Lambda \times M$;

2) проекция $\Phi:\left(\Delta_{\mathscr{B}}, \mathscr{B}\right) \rightarrow(\Lambda, T)$ является полусопряжением;

3) слои $\Phi^{-1}(z)$ являются $C^{r+1}-$ гладкими многообразиями для всех $z \in \Lambda$;

4) слои $\Phi^{-1}(z)$ гёльдерово зависят от точки $z \in \Lambda$ в $C^{r}$-норме. Более того, показатель и константа Гёльдера для n. 4) могут быть выбраны одними и теми же для всех диффеоморфизмов из $V$.

Кроме того, нам потребуется следующее утверждение, являющееся частным случаем теоремы 6.8 из [13]:

Теорема 4 (Хирш, Пью, Шуб). В условиях теоремы 3 иентральные слои непрерьвно в $C^{r+1}$-топологии зависят от точки множества $\Delta_{\mathscr{B}}$ и от диффеоморфизма $\mathscr{B}$. 
Рассмотрим отображение $\mathscr{B}: D \times S^{1} \rightarrow U\left(D^{\prime}\right) \times S^{1}, C^{1}$-близкое к $T \times \operatorname{id}_{S^{1}}$. По теореме 3 у него есть локально максимальное частично гиперболическое множество, близкое к $\Lambda \times S^{1}$ и гомеоморфное этому произведению, причем динамика на множестве центральных слоев сопряжена отображению $T: \Lambda \rightarrow \Lambda$. Более того, по теореме 4 центральные слои-окружности, на которые это множество разбивается, $C^{1}$-близки к центральным слоям невозмущенного отображения. Отображение $\mathscr{B}$ переставляет слои инвариантного множества; на множестве этих слоев возникает индуиированное отображение. Отсюда следует, что при $\mathscr{B}$, достаточно близком к $\mathscr{F}_{0}$, отображение

$$
G_{\mathscr{B}}: \Delta_{\mathscr{B}} \rightarrow \Sigma^{5} \times S^{1}, \quad G_{\mathscr{B}}(z, x)=\left(\Phi_{0} \circ \Phi(z, x), x\right),
$$

является гомеоморфизмом, $C^{1}$-гладким вдоль центральных слоев. Отметим, что $G_{\mathscr{B}}$ сохраняет координату вдоль $S^{1}$ и сопрягает индуцированное отображение на множестве центральных слоев со сдвигом Бернулли, причем координаты «вдоль базы» ( $z$ и $\omega$ соответственно) связаны взаимно гёльдеровым образом (равномерно по $x$ ). Обозначим окрестность отображения $\mathscr{F}_{0}$, в которой $G_{\mathscr{B}}$ является гомеоморфизмом, через $V_{2} \subset V$.

Запишем в этих координатах ограничение $\left.\mathscr{B}\right|_{\Delta_{\mathscr{B}}}:$ положим

$$
F_{\mathscr{B}}: \Sigma^{5} \times S^{1} \rightarrow \Sigma^{5} \times S^{1}, \quad F_{\mathscr{B}}=G_{\mathscr{B}} \circ \mathscr{B} \circ G_{\mathscr{B}}^{-1} .
$$

Мы получим мягкое гёльдерово косое произведение, $C^{1}$-гладкое вдоль слоев, отображения которого $C^{0}$-гёльдерово зависят от точки в базе. Более того, при $\mathscr{B} \in V_{2}$ это мягкое косое произведение $C^{1}$-непрерывно зависит от $\mathscr{B}$, в частности, $F_{\mathscr{B}}$ близко к тождественному отображению.

Мы будем называть $\mathscr{F}_{\mathscr{B}}$ выпрямлением отображения $\mathscr{B}$. В силу теоремы 3 найдется $C^{1}$-окрестность $V_{3} \subset V_{2}$ отображения $\mathscr{F}_{0}=T \times \mathrm{id}_{S^{1}}$ и константы $C, \alpha$, такие, что выпрямление любого из отображений $\mathscr{B} \in V_{3}$ является $(C, \alpha)$ системой. Выберем $L_{0}>1, L_{0} 2^{-\alpha}<1$.

В силу теоремы 2 в пересечении области, заданной этой теоремой, и области $V_{3}$ найдется ступенчатое косое произведение, являющееся $\left(L_{0}, C, \alpha\right)$-системой. Рассмотрим его реализацию - отображение $\mathscr{B}_{0}$. Заметим, что выпрямление $C^{1}$-малого возмущения $\mathscr{B}$ отображения $B_{0}$ будет также $\left(L_{0}, C, \alpha\right)$-системой, близкой к исходной: константа и показатель Гёльдера сохраняются в силу выбора окрестности $V_{3}$, а строгое неравенство на производные сохраняется при $C^{1}$-малом возмущении.

По построению $\mathscr{B}_{0}$ для такого выпрямления выполнено заключение теоремы 2 - наличие нулевого показателя Ляпунова вдоль слоя (поскольку это выпрямление принадлежит все той же области). Но тогда для исходного отображения $\mathscr{B}$ у соответствующей меры также один из показателей Ляпунова (а именно, центральный) равен нулю. Мы доказали, что для любого отображения $\mathscr{B}$, достаточно $C^{1}$-близкого к $\mathscr{B}_{0}$, найдется эргодическая инвариантная мера, один из показателей Ляпунова которой равен нулю.

Наконец, как несложно видеть, само отображение $\mathscr{B}_{0}$ может быть продолжено до диффеоморфизма любого трехмерного многообразия (поскольку оно легко продолжается до диффеоморфизма полнотория $[-1,2] \times[-1,2] \times S^{1}$, тождественного на границе, а полноторие можно вложить в любое трехмерное многообразие). Поскольку у близких диффеоморфизмов близки и их ограничения на $D \times S^{1}$, теорема 1 тем самым доказана. 


\section{§6. Выбор области в пространстве мягких косых произведений и расхождение траекторий}

Пусть $a-$ произвольное конечное слово из символов $0, \ldots, 4$. Через $\{\ldots \mid a \ldots\}$ мы обозначаем произвольную бесконечную последовательность $\omega \in \Sigma^{5}$, в которой, начиная с нулевого места, записано слово $a$. Аналогично вводим обозначения $\{\ldots a \mid \ldots\}$ и $\{\ldots a \mid b \ldots\}$. В дальнейшем мы будем применять греческую букву $\omega$ для обозначения бесконечных, а латинскую $w$ для конечных слов. Наконец, для заданного конечного слова $w$ (с указанием нулевого места) через $(w)$ мы обозначим бесконечную периодическую последовательность с периодом $w$ (соответствующим образом расположенным).

Определение 4. Пусть мягкое косое произведение $F$ является $(L, C, \alpha)$ системой в смысле определения 2. Скажем, что оно обладает свойством

- растяжения, если существуют $\nu>1, \delta_{1}>0$, такие, что для произвольного интервала $I \subset S^{1},|I|<\delta_{1}$,

$$
\exists j_{1} \in\{0, \ldots, 4\}: \forall \omega=\left\{\ldots \mid j_{1} \ldots\right\} \forall x \in I \quad\left(D f_{\omega}\right)(x)>\nu
$$

- обратного растяжения, если существуют $\nu>1, \delta_{1}>0$, такие, что для произвольного интервала $I \subset S^{1},|I|<\delta_{1}$,

$$
\exists j_{2} \in\{0, \ldots, 4\}: \forall \omega=\left\{\ldots j_{2} \mid \ldots\right\} \forall x \in I \quad\left(D f_{\sigma^{-1} \omega}^{-1}\right)(x)>\nu ;
$$

- наличия $\delta_{2}$-поворота, если для любой последовательности $\omega=\{\ldots \mid 0 \ldots\}$

$$
d_{C^{0}}\left(f_{\omega}, R_{\delta_{2}}\right)<\frac{\delta_{2}^{2}}{40}
$$

здесь через $R_{\delta_{2}}$ обозначен поворот на угол $\delta_{2}$;

- наличия слабо притягивающей орбиты, если существует притягивающая периодическая орбита $X$, для показателя Ляпунова вдоль слоя которой, обозначаемого $\lambda(X)<0$,

$$
\lambda(X)+\ln \nu>0
$$

- $\gamma$-предсказуемости траекторий, $\gamma>0$, если для любой точки $x \in$ $S^{1}$, для любого натурального $m$ и для любого конечного слова $w^{*}=$ $\left\{w_{-m} \ldots w_{-1} \mid w_{0} \ldots w_{m-1}\right\}$

$$
\begin{aligned}
\operatorname{diam}\left\{\bar{f}_{m}[\omega](x) \mid \omega\right. & \left.=\left\{\ldots w^{*} \ldots\right\}\right\}<\gamma, \\
\operatorname{diam}\left\{\bar{f}_{-m}[\omega](x) \mid \omega\right. & \left.=\left\{\ldots w^{*} \ldots\right\}\right\}<\gamma .
\end{aligned}
$$

Наконец, будем говорить, что система обладает свойством управляемости, или просто управляема, если для нее имеют место все описанные выше свойства, причем константы могут быть выбраны удовлетворяющими условию согласованности констант. Последнее состоит в том, что

$$
\gamma<\delta_{2} / 40, \quad \delta_{1}>3 \delta_{2}
$$

$$
\alpha>\log _{2} L
$$


Ясно, что все эти свойства, кроме свойства предсказуемости траекторий, могут быть выполнены одновременно для соответствующим образом подобранного ступенчатого косого произведения, а также для всех достаточно близких к нему мягких систем. Следующая лемма, принадлежащая А. С. Городецкому, выводит предсказуемость траекторий из условия на показатель Гёльдера и скорость растяжения.

Лемма 1 [15, лемма 3.1]. Пусть заданы L, $C$ и $\alpha$, такие, что выполнено условие $\alpha>\log _{2} L$ - второе из условий согласованности констант. Тогда существует такое $K=K(L, C, \alpha)$, что для любой $(L, C, \alpha)$-системь, $\delta$-близкой $\kappa$ ступенчатой,

$$
d_{C_{0}}\left(\bar{f}_{ \pm m}[\omega], \bar{f}_{ \pm m}\left[\omega^{\prime}\right]\right) \leqslant \gamma:=K \delta^{\beta},
$$

әде $\beta=1-\left(\ln L / \ln 2^{\alpha}\right)$.

\section{§7. Основная лемма о периодических орбитах}

Определение 5. Пусть задана периодическая орбита $X$ отображения $F$, период этой орбиты равен $P$ и $\varepsilon>0$ фиксировано. Точка $y$ называется $(\varepsilon, P)-x o$ рошей для орбиты $X$, если найдется точка $x \in X$, такая, что

$$
\forall l=0,1 \ldots P-1 \quad d\left(F^{l}(x), F^{l}(y)\right)<\varepsilon .
$$

Лемма 2 (основная лемма). Пусть косое произведение $F$ вида (2) обладает свойством управляемости. Пусть $X$ - его произвольная периодическая орбита периода $P$ с мультипликатором по слою $\theta, 0<\theta<1$, для показателя Ляпунова вдоль слоя $\lambda:=\ln \theta / P$ которой выполняется неравенство

$$
\lambda+\ln \nu>0 \text {. }
$$

Тогда для любого $\varepsilon>0$ существует периодическая орбита $Y$ косого произведения $F$ с периодом $P^{\prime}>2 P$ и показателем Ляпунова вдоль слоя $\lambda^{\prime}<0$, такая, чmo

1) $\left|\lambda^{\prime}\right|<C|\lambda|$, где $C=C(F)$ - глобальная константа, зависящая только от косого произведения $F, 0<C<1$;

2) $\lambda^{\prime}+\ln \nu>0$;

3) существует $\tilde{Y} \subset Y$ и проекиия $\pi: \widetilde{Y} \rightarrow X$, такие, что

(а) все точки множества $\widetilde{Y}$ являются $(\varepsilon, P)$-хорошими относительно орбиты $X$, причем в определении 5 можно взять $x=\pi(y)$,

(b) доля $\varkappa:=\# \widetilde{Y} / \# Y$ точек, в которых определена проекиия $\pi$, допускает оценку снизу:

$$
\varkappa \geqslant 1-\frac{3|\lambda|}{\ln L} ;
$$

(с) количество элементов прообраза $\pi^{-1}(x)$ одинаково для всех $x \in X$.

Периодическая орбита косого произведения задается своей начальной точкой $(\omega, x), \omega \in \Sigma^{N}, x \in S^{1}, \omega=(w)$ - периодическая последовательность, $w=$ $\left\{\mid w_{0} \ldots w_{P-1}\right\}-$ ее период и

$$
\sigma^{P} \omega=\omega, \quad \bar{f}_{P}[\omega](x)=x .
$$

Идея доказательства - зафиксировав интервал $J$ на центральном слое, подобрать серию слов $w^{\prime}(k)$ в базе, длина которых возрастает с ростом натурального 
параметра $k$. Соответствующие отображения косого произведения будут сжимать $J$ в себя, гарантируя наличие притягивающей вдоль слоя неподвижной точки, а их производная на $J$ будет равномерно ограничена по $k$. Увеличивая $k$, можно добиться сколь угодно близкого к нулю отрицательного показателя Ляпунова.

Попутно, строя новую орбиту $Y$, мы обеспечиваем близость точек новой и старой орбит (свойства $3(\mathrm{a})-3(\mathrm{c})$ ). Это позволит нам получить эргодичность предельной меры как следствие теоремы Биркгофа-Хинчина.

Доказательство леммы 2. Пусть задана периодическая орбита $X$ с начальной точкой $(\omega, x)=((w), x)$, где $w$ - период последовательности $\omega$. Мы будем строить новую периодическую орбиту $Y$ с начальной точкой $\left(\omega^{\prime}, y\right)=\left(\left(w^{\prime}\right), y\right)$, где

$$
w^{\prime}=\left\{R_{1} w^{n_{1}} w^{r^{\prime}(k)} w^{k} \mid w^{k} w^{n_{1}} R_{2} R_{3}\right\} .
$$

Здесь $k, r^{\prime}(k), n_{1}$ - большие натуральные числа, которые будут выбраны ниже, $w, w^{\prime}, R_{i}$ - слова конечной длины, $w^{m}$ есть $m$ раз повторенное слово $w$, вертикальная черта отмечает место в периоде, соответствующее началу отсчета в $\omega^{\prime}$, и, наконец, $x, y-$ точки на окружности.

Слова $w^{k}$ в (12) обеспечивают одновременно неограниченное увеличение длины слова $w^{\prime}$ и сильное сжатие некоторого интервала $J$ до мельчайшего размера; слово $R_{2}$ растягивает образ интервала $J$ до $J_{2}$, используя меньшее количество букв (с удельной эффективностью, т. е. с логарифмом производной в расчете на одну букву, по меньшей мере, $\ln \nu$ вместо близкого к нулю $|\lambda|$ для букв орбиты $X$ ); слово $R_{1}$ растягивает прообраз интервала $J$ до $J_{1}$ при обратных итерациях, создавая тем самым «воронку», попадание в (большую) «горловину» которой гарантирует попадание в $J$ через соответствующее число итераций; слово $R_{3}$ отображает интервал $J_{2}$ внутрь $J_{1}$, замыкая цепочку включений, так что построенное периодическое слово за период отображает $J$ в себя; слова $w^{n_{1}}$ и $w^{r^{\prime}(k)}$ несут техническую функцию - позволяют контролировать «погрешность» вдоль слоя.

Зафиксируем $\varepsilon>0$. В силу Diff ${ }^{1}$-непрерывной зависимости $f_{\omega}$ от параметра в базе, для любых $\theta^{+}, \theta^{-}, 0<\theta^{-}<\theta<\theta^{+}<1$, найдутся число $n_{1}\left(\theta^{ \pm}\right) \in \mathbb{N}$ и интервал $J\left(n_{1}, \theta^{ \pm}\right) \subset S^{1}, J \ni x, L^{P}|J|<\varepsilon,|J|<\delta_{1}$, такие, что для любой последовательности $\omega^{*}=\left\{\ldots w^{n_{1}} \mid w^{n_{1}} \ldots\right\}$

$$
\begin{gathered}
\bar{f}_{P}\left[\omega^{*}\right](J) \subset J, \\
\left.D \bar{f}_{P}\left[\omega^{*}\right]\right|_{J} \in\left(\theta^{-}, \theta^{+}\right)
\end{gathered}
$$

и для всех $l, 0 \leqslant l \leqslant P-1$, и $y \in J$

$$
\operatorname{diam}\left\{\bar{f}_{l}\left[\omega^{*}\right](y) \mid \omega^{*}=\left\{\ldots w^{n_{1}} \mid w^{n_{1}} \ldots\right\}\right\}<\varepsilon .
$$

Ограничение сверху на длину $J$ немедленно влечет за собой неравенство

$$
\rho\left(\bar{f}_{l}[\omega](x), \bar{f}_{l}[\omega](y)\right)<\varepsilon
$$

для любой пары точек $x, y \in J$, произвольного целого $l=0, \ldots, P-1$ и фиксированного $\omega$.

Выберем $\theta^{-}$и $\theta^{+}$достаточно близкими к $\theta$ (конкретные требования на них будут указаны позднее) и зафиксируем $n_{1}\left(\theta^{ \pm}\right)$и $J\left(n_{1}, \theta^{ \pm}\right)$.

Все константы и интервалы, которые будут построены ниже, естественно, зависят от выбора $J, n_{1}$ и $\theta^{ \pm}$; мы не будем далее подчеркивать эту зависимость. 
Предложение 1 («воронка»). Найдутся конечное слово $R_{1}$ и интервал $J_{1}$ длины $\left|J_{1}\right| \geqslant \delta_{1}-4 \gamma$, такие, что для всех $\omega=\left\{\ldots R_{1} w^{n_{1}} \mid w^{n_{1}+\left\lceil\left|R_{1}\right| / P\right\rceil} \ldots\right\}$

$$
\bar{f}_{-\left(\left|R_{1}\right|+n_{1} P\right)}[\omega](J) \supset J_{1} .
$$

Доказательство. Будем строить слово $R_{1}$ по индукции, начиная с пустого слова и выписывая его буквы справа налево. Пусть последние $l$ букв, составляющие слово $R_{1}(l)$, уже построены. Рассмотрим какое-нибудь слово $\omega$ вида $\left\{\ldots R_{1}(l) w^{n_{1}} \mid w^{n_{1}+\lceil l / P\rceil} \ldots\right\}$ и найдем прообраз интервала $I_{l}=\bar{f}_{-\left(l+n_{1} P\right)}[\omega](J)$.

Если длина интервала $I_{l}$ превосходит $\delta_{1}-2 \gamma$, то, применив к концам интервала $J$ свойство предсказуемости траекторий (9), видим, что для любого другого слова $\omega$ того же вида $\bar{f}_{-\left(l+n_{1} P\right)}[\omega](J) \supset J_{1}$, где интервал $J_{1}$ получается из $I_{l}$ отступлением внутрь на $\gamma$ от каждого конца. Требуемый интервал $J_{1}$ и слово $R_{1}=R_{1}(l)$ построены.

В случае же, если длина интервала $I_{l}$ меньше $\delta_{1}-2 \gamma$, опять-таки из свойства предсказуемости траекторий следует, что для любого другого слова $\omega$ того же вида

$$
\bar{f}_{-\left(l+n_{1} P\right)}[\omega](J) \subset I_{l}^{*}:=U_{\gamma}\left(I_{l}\right) .
$$

Поскольку $\left|I_{l}^{*}\right|<\delta_{1}$, в силу свойства растяжения в обратном времени найдется буква $a_{l+1} \in\{0, \ldots, 4\}$, такая, что все соответствующие ей отображения растягивают в каждой точке интервала $I_{l}^{*}$ при взятии прообраза по меньшей мере в $\nu$ раз. Ее мы и допишем в начало: $R_{1}(l+1)=a_{l+1} R_{l}$.

Легко видеть, что такой процесс оборвется не позже, чем через $C=$ $\left\lceil\log _{\nu}\left(\delta_{2} L^{n_{1} P} /|J|\right)\right\rceil$ шагов, поскольку за каждый из первых $n_{1} P$ шагов прообраз уменьшается в не более чем $L$ раз, а за каждый из последующих возрастает по крайней мере в $\nu$ раз.

Отметим, что из вложения (17) следует вложение

$$
\bar{f}_{\left|R_{1}\right|+n_{1} P}[\omega]\left(J_{1}\right) \subset J
$$

для любой последовательности $\omega=\left\{\ldots \mid R_{1} w^{2 n_{1}+\lceil l / P\rceil} \ldots\right\}$.

Предложение 2 («поворот»). Для любого интервала $J_{2}$ длинъ $\left|J_{2}\right|<\delta_{2}$ найдется некоторое $j, 0 \leqslant j \leqslant M:=\left\lceil 1 / \delta_{2}\right\rceil$, такое, что для любого слова $\omega=\left\{\ldots \mid 0^{j} \ldots\right\}$

$$
\bar{f}_{j}[\omega]\left(J_{2}\right) \subset J_{1} .
$$

(Положим $R_{3}:=0^{j}$.)

Доказательство. Заметим сначала, что если отображения $f_{1}, \ldots, f_{j} \bar{\delta}$-близки в метрике $C^{0}$ к повороту $R_{\delta_{2}}$, то их композиция $j \bar{\delta}$-близка к повороту $R_{j \delta_{2}}$ :

$$
\begin{aligned}
& d\left(f_{1} \circ \cdots \circ f_{j}, R_{\delta_{2}}^{j}\right) \leqslant d\left(f_{1} \circ \cdots \circ f_{j}, R_{\delta_{2}} \circ f_{2} \circ \cdots \circ f_{j}\right) \\
& \quad+d\left(R_{\delta_{2}} \circ f_{2} \circ \cdots \circ f_{j}, R_{\delta_{2}}^{2} \circ f_{3} \circ \cdots \circ f_{j}\right)+\cdots+d\left(R_{\delta_{2}}^{j-1} \circ f_{j}, R_{\delta_{2}}^{j}\right) \\
& \quad \leqslant d\left(f_{1}, R_{\delta_{2}}\right)+\cdots+d\left(f_{j}, R_{\delta_{2}}\right) \leqslant j \bar{\delta} .
\end{aligned}
$$

Как следует из несложных геометрических соображений, найдется $j, 0 \leqslant j \leqslant M$, такое, что поворот на угол $j \delta_{2}$ перемещает $J_{2}$ внутрь $J_{1}$ так, что оба интервала дополнения имеют длину, не меньшую $\delta_{2} / 2$ (напомним, что $\delta_{1}>3 \delta_{2}$ ). 
Тогда для любого слова $\omega=\left\{\ldots \mid 0^{j} \ldots\right\}$ в силу сказанного выше

$$
\bar{f}_{j}[\omega]\left(J_{2}\right) \subset U_{j \cdot \delta_{2}^{2} / 40}\left(R_{\delta_{2}}^{j}\left(J_{2}\right)\right) \subset U_{\delta_{2} / 5}\left(R_{j \delta_{2}}\left(J_{2}\right)\right) \subset J_{1} .
$$

Фиксируем $C$ и $M$ согласно предложениям 1 и 2 . Положим

$$
\delta=\min \left(\frac{1}{L^{2 n_{1}\left(J, \theta^{ \pm}\right) P+C(J)+M}}, \frac{\delta_{2}-2 \gamma}{L^{n_{1} P}|J|}\right)
$$

Пусть $k$ - произвольное большое число, $k>\max (C(J), M)$ (далее мы будем накладывать на $k$ дополнительные ограничения снизу).

Выберем $r(k)$ так, чтобы выполнялось неравенство

$$
\frac{\delta}{L} \leqslant\left(\theta^{+}\right)^{k} L^{r(k)}<\delta .
$$

Положим $r^{\prime}(k):=[r(k) / P]+1$.

Предложение 3 («растяжение»). Найдутся слово $R_{2}=R_{2}(k)$ длины $\left|R_{2}\right|=$ $r(k)$ и интервал $J_{2}=J_{2}(k)$ длины, не большей $\delta_{2}$, такие, что для любой последовательности $\omega=\left\{\ldots w^{n_{1}+r^{\prime}(k)+k} \mid w^{k+n_{1}} R_{2} \ldots\right\}$

$$
\begin{gathered}
\left.D \bar{f}_{S+r(k)}[\omega]\right|_{J}>\left(\theta^{-}\right)^{k} L^{-n_{1} P} \nu^{r(k)}, \\
\bar{f}_{S+r(k)}[\omega](J) \subset J_{2},
\end{gathered}
$$

где $S:=\left(k+n_{1}\right) P$.

Доказательство. Как и в предложении 1 , мы выписываем слово $R_{2}$ по индукции, начиная с пустого слова. Считая, что первые $l$ букв, составляющие слово $R_{2}^{*}(l)$, уже написаны, мы рассматриваем $(l+S)$-й образ $I_{l}$ интервала $J$ для какого-нибудь слова $\omega=\left\{\ldots w^{n_{1}+r^{\prime}(k)+k} \mid w^{k+n_{1}} R_{2} \ldots\right\}$ :

$$
I_{l}=\bar{f}_{l+S}[\omega](J) .
$$

Заметим, что этот образ имеет длину, не большую

$$
|J| \cdot\left(\theta^{+}\right)^{k} \cdot L^{n_{1} P+l} \leqslant\left(|J| L^{n_{1} P}\right) \cdot\left(\theta^{+}\right)^{k} \cdot L^{r(k)} \leqslant \delta_{2}-2 \gamma .
$$

Значит (в силу свойства предсказуемости траекторий (8)), $l$-й образ интервала $J$ под действием любого другого слова $\omega$ того же вида содержится в соответствующей $\gamma$-окрестности $I_{l}^{*}:=U_{\gamma}\left(I_{l}\right)$; при этом $\left|I_{l}^{*}\right| \leqslant 2 \gamma+\left|I_{l}\right| \leqslant \delta_{2}$. В силу свойства растяжения (4) мы можем выбрать букву $b_{l+1}$, такую, что все соответствующие ей отображения растягивают на $I_{l}^{*}$ по крайней мере в $\nu$ раз. Допишем эту букву в конец уже построенной части: $R_{2}^{*}(l+1)=R_{2}^{*}(l) b_{l+1}$. Выписав $r(k)$ букв, мы останавливаем процесс и полагаем $R_{2}=R_{2}^{*}(r(k))$. Тогда, как следует из сказанного выше,

$$
\forall \omega=\left\{\ldots w^{n_{1}+r^{\prime}(k)+k} \mid w^{k+n_{1}} R_{2} \ldots\right\} \quad \bar{f}_{S+r(k)}[\omega](J) \subset J_{2}:=I_{r(k)}^{*} .
$$

С другой стороны, поскольку у каждого из применяемых на участке $R_{2}$ отображений производная на соответствующем интервале не меньше $\nu$, получаем

$$
\left.D \bar{f}_{S+r(k)}[\omega]\right|_{J}>\left(\theta^{-}\right)^{k} L^{-n_{1} P} \nu^{r(k)} .
$$

Выберем и зафиксируем $R_{1}$ и $J_{1}$ по предложению 1 , а $R_{2}$ и $J_{2}$ - по предложению 3 . Выберем $R_{3}$ по предложению 2. 
Рассмотрим периодическую последовательность $\omega^{\prime}$, задаваемую словом

$$
w^{\prime}=\left\{R_{1} w^{n_{1}+r^{\prime}(k)+k} \mid w^{k+n_{1}} R_{2} R_{3}\right\} .
$$

Легко видеть, что в силу цепочки включений (23), (19), (18) и (13) (последнее применяется $k+r^{\prime}(k)$ раз) имеем

$$
\bar{f}_{\left|w^{\prime}\right|}\left[\omega^{\prime}\right](J) \subset J .
$$

Из (20) и (21) следует оценка

$$
\left.\left(D \bar{f}_{\left|w^{\prime}\right|}\left[\omega^{\prime}\right]\right)\right|_{J} \leqslant\left(\theta^{+}\right)^{2 k+r^{\prime}(k)} L^{2 n_{1} P+r(k)+M+C(J)}<1 ;
$$

поэтому отображение $\bar{f}_{\left|w^{\prime}\right|}\left[\omega^{\prime}\right]$ обладает на интервале $J$ притягивающей неподвижной точкой. Обозначим ее через $y$. Орбита $Y=\left(\omega^{\prime}, y\right)$ построена.

Предложение 4. При достаточно больших $k$ показатель Ляпунова орбиты $Y$ обладает свойствами 1) и 2) из леммы 2.

Доказательство. Напомним, что мы до сих пор не установили требования на $\theta^{ \pm}$в построении орбиты $Y$. Они будут конкретизированы в доказательстве данного предложения.

Оценим снизу производную отображения $\bar{f}_{\left|w^{\prime}\right|}\left[\omega^{\prime}\right]$ на интервале $J$ :

$$
\left.\left(D \bar{f}_{\left|w^{\prime}\right|}\left[\omega^{\prime}\right]\right)\right|_{J} \geqslant\left(\theta^{-}\right)^{2 k+r^{\prime}(k)} \nu^{r(k)} L^{-\left(2 n_{1} P+M+C(J)\right)} .
$$

По (21) найдется константа $C_{1}=C_{1}\left(J, \theta^{ \pm}, n_{1}\right)$, не зависящая от $k$, такая, что

$$
r(k)>\frac{1}{\ln L}\left(-k \ln \theta^{+}\right)+C_{1},
$$

откуда найдутся константы $C_{2}$ и $C_{3}$, также не зависящие от $k$, для которых

$$
\begin{aligned}
\ln \left\|D \bar{f}_{\left|w^{\prime}\right|}\left[\omega^{\prime}\right]\right\| & \geqslant\left(2 k+\frac{r(k)}{P}\right) \ln \theta^{-}+r(k) \ln \nu+C_{2} \\
& >2 k \ln \theta^{-}-\frac{k \ln \theta^{+} \ln \theta^{-}}{P \ln L}+\frac{C_{1} \ln \theta^{-}}{P \ln L}-\frac{k \ln \theta^{+} \ln \nu}{\ln L}+C_{3},
\end{aligned}
$$

что влечет за собой оценку

$$
\lambda^{\prime}:=\frac{\ln \left\|D \bar{f}_{\left|w^{\prime}\right|}\left[\omega^{\prime}\right](y)\right\|}{\left|w^{\prime}\right|}>\frac{\ln \left\|D \bar{f}_{\left|w^{\prime}\right|}\left[\omega^{\prime}\right](y)\right\|}{2 k P}=h(\alpha)+O\left(\frac{1}{k}\right)
$$

для показателя Ляпунова орбиты $Y$, где

$$
h(\alpha):=\lambda\left((1-\alpha)-\frac{\left(1-\alpha^{2}\right) \ln \theta}{2 P \ln L}-\frac{(1+\alpha) \ln \nu}{2 \ln L}\right), \quad \theta^{ \pm}:=\theta^{1 \pm \alpha}, \alpha>0 .
$$

Функция $h(\alpha)$ непрерывна в нуле; кроме того, в силу неравенств

$$
0<q:=\frac{\lambda+\ln \nu}{2 \ln L}<1
$$

выполняется оценка

$$
h(0)=\lambda\left(1-\frac{\ln \theta / P+\ln \nu}{2 \ln L}\right)>(1-q) \lambda .
$$


Выберем и зафиксируем $\alpha<1$ (а с ним и $\theta^{ \pm}$) таким малым, чтобы выполнялась оценка $h(\alpha)>(1-q / 2) \lambda$. Тогда при достаточно больших $k$ оценка

$$
\lambda^{\prime}>(1-q / 3) \lambda,
$$

доказывает свойство 1) из леммы 2.

Неравенство $\lambda^{\prime}>\lambda$ влечет за собой свойство 2).

Осталось обосновать свойство 3 ) из леммы 2. Определим множество $\widetilde{Y}$ и проекцию $\pi$ следующим образом. Пусть $K=K(\varepsilon, w)$ - минимальное натуральное число, такое, что $2^{-K P} \leqslant \varepsilon$. При $k>K$ положим

$$
\widetilde{Y}=\left\{F^{j}\left(\omega^{\prime}, x^{\prime}\right) \mid-(k-K) P \leqslant j<(k-K-1) P\right\}
$$

Зададим проекцию $\pi: \widetilde{Y} \rightarrow X$ формулой

$$
\pi\left(F^{j}\left(\omega^{\prime}, x^{\prime}\right)\right)=F^{\rho}(\omega, x),
$$

где $\rho$ - остаток от деления числа $j$ на $P$. Очевидно, число точек в прообразе $\pi^{-1}(\tilde{\omega}, \tilde{x})$ не зависит от $(\tilde{\omega}, \tilde{x})$ и равно $k-2 P-1$. Утверждение $3($ с) леммы выполнено.

Предложение 5. Все точки множества $\widetilde{Y}$ являются $(3 \varepsilon, P)$-хорошими для орбитъ $X$.

Доказательство. Оценим расстояние по базе. В силу выбора $K$ для любой точки $\tilde{y} \in \tilde{Y}$ расстояние между $\Sigma^{N}$-координатами точек $F^{l}(\tilde{y}) \in \widetilde{Y}$ и $\pi\left(F^{l}(\tilde{y})\right)$ $\in X$ не превосходит $\varepsilon$ для всех $l=0, \ldots, P-1$.

Оценим расстояние по слою. По построению точка $x^{\prime}$ после итераций на участке $w^{k+n_{1}} R_{2} R_{3} R_{1} w^{n_{1}}$ оказывается внутри интервала $J$. Следующие итерации в силу выбора $n_{1}$ и соотношения (13) отображают $J$ в себя. Соответственно расстояние между $F^{j}\left(\omega^{\prime}, x^{\prime}\right)$ и $F^{j}(\omega, x)=F^{\rho}(\omega, x)$ есть расстояние между $\rho$-ми итерациями некоторых точек из $J$ под действием некоторых (разных!) слов, имеющих вид $\left\{\ldots w^{n_{1}} \mid w^{n_{1}} \ldots\right\}$, где $\rho$ - остаток от деления числа $j$ на $P$.

Будем итерировать точку, соответствующую новой орбите, словом $\omega$ (координата по базе старой орбиты). В силу неравенства (15) изменение координаты вдоль слоя за $\rho$ итераций не превосходит $\varepsilon$. Но теперь обе точки итерируются словом старой орбиты, а число итераций не превосходит $P$; значит, в силу неравенства (16) расстояние между ними не больше $\varepsilon$. Итого, расстояние по слою между $F^{j}\left(\omega^{\prime}, x^{\prime}\right)$ и $F^{j}(\omega, x)$ не превосходит $2 \varepsilon$.

Отсюда следует, что первые $P$ итераций точек $\tilde{y} \in \widetilde{Y}$ и $\pi(\tilde{y})$ расходятся в $\Sigma^{N} \times S^{1}$ на расстояние меньше $3 \varepsilon$. Это доказывает утверждение 3(а) леммы 2.

Оценим долю точек на орбите $Y$, не являющихся хорошими для орбиты $X$. Найдется константа $C_{4}$, не зависящая от $k$, такая, что

$$
1-\frac{\# \tilde{Y}}{\# Y}=\frac{2 n_{1} P+r^{\prime}(k) P+r(k)+M+C(J)+(2 K+1) P}{\left|w^{\prime}\right|} \leqslant \frac{2 r(k)+C_{4}}{2 k P} .
$$

В силу (21) найдется константа $C_{5}$, не зависящая от $k$, такая, что

$$
r(k)<\frac{1}{\ln L}\left(-k \ln \theta^{+}\right)+C_{5}<\frac{1}{\ln L}(-2 k \ln \theta)+C_{5},
$$


а значит,

$$
1-\frac{\# \tilde{Y}}{\# Y}<-\frac{2 \lambda}{\ln L}+O\left(\frac{1}{k}\right) .
$$

Отсюда следует утверждение 3(b) леммы 2 для достаточно больших $k$. Лемма 2 полностью доказана.

\section{§8. Последовательность периодических орбит, эргодичность и нулевые показатели Ляпунова}

В этом параграфе мы формулируем лемму о нулевом показателе Ляпунова и доказываем теорему 2.

Лемма 3. Для любой управляемой системы найдется эргодическая инвариантная мера с нулевым показателем Ляпунова вдоль слоя.

Доказательство. Воспользовавшись леммой 2, мы можем построить последовательность притягивающих вдоль слоя периодических орбит, начинающуюся со слабо притягивающей орбиты (см. (7)). Показатели Ляпунова для этих орбит экспоненциально стремятся к нулю, причем следующая орбита бо́льшую часть времени проводит около предыдущей.

Рассмотрим последовательность атомарных мер, равномерно распределенных на этих орбитах. Из условия на «похожесть» орбит (см. утверждение 3 ) леммы 2) с помощью эргодической теоремы Биркгофа-Хинчина выводится, что любая предельная точка построенной последовательности будет эргодической инвариантной мерой; также несложно проверяется неатомарность предельной меры. Поскольку пространство мер на $\Sigma^{5} \times S^{1}$ *-слабо компактно, мы можем выделить из этой последовательности сходящуюся - в силу сказанного выше к эргодической инвариантной мере - подпоследовательность. Эта предельная мера и будет искомой.

Действительно, показатель Ляпунова для эргодической инвариантной меры выражается как интеграл по этой мере от непрерывной функции - производной отображения вдоль слоя. Поэтому показатель Ляпунова для предельной меры равен пределу показателей Ляпунова, т. е. нулю.

Эти рассуждения строго проведены в работе [18] (см. леммы 1 и 2) для случая ступенчатых систем и дословно переносятся на мягкий случай. Поэтому мы их не будем здесь повторять в полном объеме.

Доказательство теоремы 2. В силу леммы 4 (см. §9) существует открытая область в пространстве $(L, C, \alpha)$-систем, содержащая ступенчатые системы, сколь угодно близкие к тождественной (вдоль слоя), и такая, что каждое отображение из этой области управляемо. С другой стороны, в силу леммы 3 для каждой управляемой системы найдется эргодическая мера с нулевым показателем Ляпунова вдоль слоя.

Таким образом, любое отображение из построенной области обладает эргодической мерой с нулевым показателем Ляпунова вдоль слоя. Теорема 2 доказана.

\section{§9. Построение областей управляемых систем}

В этом разделе мы для любых заданных $L>1, C>0$ и $\alpha \in(0,1)$ построим сколь угодно близкие к тождественным диффеоморфизмы окружности $g_{0}, g_{1}, \ldots, g_{4}$, такие, что все $(L, C, \alpha)$-системы, близкие к соответствующему 
ступенчатому произведению, будут управляемыми. Эта конструкция завершает доказательство теоремы 2, а тем самым и основного результата, теоремы 1.

Пусть константы $L, C$ и $\alpha$ заданы. Выберем $L_{1}<L, 1<L_{1}<2^{\alpha}$. Все диффеоморфизмы $g_{i}$, которые будут построены ниже, будут выбраны так, чтобы удовлетворялось ограничение на производные

$$
\max _{x \in S^{1}} \max \left(g_{i}^{\prime}(x),\left(g_{i}^{-1}\right)^{\prime}(x)\right)<L_{1} .
$$

Пусть $W \subset \operatorname{Diff}^{1}\left(S^{1}\right)$ - произвольно малая окрестность тождественного отображения окружности. Представим окружность $S^{1}$ в виде $S^{1}=\mathbb{R} / \mathbb{Z}$, и пусть $g_{1}: S^{1} \rightarrow S^{1}$ - диффеоморфизм Морса-Смейла с аттрактором $p=0$ и репеллером $q=1 / 2, g_{1}^{\prime}(x)<1$ при $x \in[-1 / 5,1 / 5], g_{1}^{\prime}(x)>1$ при $x \in[3 / 10,7 / 10]$, принадлежащий $W$. Положим $g_{2}(x):=g_{1}(x+1 / 3), g_{3}(x):=g_{1}(x-1 / 3)$.

Положим

$$
\delta_{1}:=\frac{1}{15}, \quad \nu_{0}:=\min \left(\left(\max _{x \in[-1 / 5 \ldots 1 / 5]} g_{1}^{\prime}(x)\right)^{-1}, \min _{x \in[3 / 10 \ldots 7 / 10]} g_{1}^{\prime}(x)\right) .
$$

Тогда для любого интервала $I$ длины меньше $\delta_{1}$ в каждой точке одно из отображений $g_{i}(i=1,2,3)$ растягивает его с производной не менее $\nu_{0}$. То же верно и для обратных отображений.

Зафиксируем $\delta_{2}, 0<\delta_{2}<\delta_{1} / 3$, так, чтобы отображение $g_{0}:=R_{\delta_{2}}$ (поворот окружности на угол $\left.\delta_{2}\right)$ принадлежало $W$.

Пусть $g_{4} \in W$ - диффеоморфизм Морса-Смейла с притягивающей периодической орбитой $X_{0}$, для показателя Ляпунова которой выполняется неравенство

$$
\lambda\left(X_{0}\right)+\frac{\ln \nu_{0}}{2}>0 .
$$

Лемма 4. В достаточно малой окрестности ступенчатой системы, соответствующей построенным отображениям $g_{0}, \ldots, g_{4}$, в пространстве $(L, C, \alpha)$ систем все системы являются управляемыми.

Доказательство. Все соответствующие условия, кроме предсказуемости траекторий, сохраняются при малом возмущении; кроме того, они по построению выполнены для самой ступенчатой системы. Предсказуемость траекторий получается как следствие второй части условия согласованности констант и леммы 1. Для выполнения первой части условия согласованности констант достаточно потребовать выполнения неравенства $K \delta^{\beta}<\delta_{2} / 40$ для расстояния $\delta$ от ступенчатой системы до ее возмущения.

Все требования, составляющие условие управляемости, выполнены в достаточно малой окрестности построенной ступенчатой системы.

\section{ЛИТЕРАТУРА}

[1] R. Abraham, S. Smale, Nongenericity of $\Omega$-stability, in: Global Analysis (Proc. Sympos. Pure Math., Vol. XIV, Berkeley, Calif., 1968), Amer. Math. Soc., Providence, R.I., 1970, 5-8.

[2] Я. Б. Песин, Характеристические показатели Ляпунова и гладкая эргодическая теория, УМН, 32(196):4 (1977), 55-112.

[3] H. Furstenberg, H. Kesten, Products of random matrices, Ann. Math. Statist., 31 (1960), 457-469. 
[4] C. Bonatti, X. Gomez-Mont, M. Viana, Généricité d'exposants de Lyapunov nonnuls pour des produits déterministes de matrices, Ann. Inst. H. Poincaré, Anal. Non Linéaire, 20:4 (2003), 579-624.

[5] L. Arnold, N. D. Cong, V. I. Oseledets, Jordan normal form for linear cocycles, Random Oper. Stochastic Equations, 7:4 (1999), 303-358.

[6] L. Arnold, N. D. Cong, Linear cocycles with simple Lyapunov spectrum are dense in $L^{\infty}$, Ergodic Theory Dynam. Systems, 19:6 (1999), 1389-1404.

[7] J. Bochi, Genericity of zero Lyapunov exponents, Ergodic Theory Dynam. Systems, 22:6 (2002), 1667-1696.

[8] J. Bochi, M. Viana, Uniform (projective) hyperbolicity or no hyperbolicity: a dichotomy for generic conservative maps, Ann. Inst. H. Poincaré, Anal. Non Linéaire, 19:1 (2002), 113-123.

[9] A. Baraviera, C. Bonatti, Removing zero Lyapunov exponents, Ergodic Theory Dynam. Systems, 23 (2003), 1655-1670.

[10] M. Shub, A. Wilkinson, Pathological foliations and removable zero exponents, Invent. Math., 139 (2000), 495-508.

[11] D. Ruele, A. Wilkinson, Absolutely singular dynamical foliations, Comm. Math. Phys., 219:3 (2001), 481-487.

[12] D. Dolgopyat, Y. Pesin, Every compact manifold carries a completely hyperbolic diffeomorphism, Ergodic Theory Dynam. Systems, 22:2 (2002), 409-435.

[13] M. W. Hirsch, C. C. Pugh, M. Shub, Invariant Manifolds, Lecture Notes in Math., vol. 583, Springer-Verlag, Berlin-New York, 1977.

[14] А. С. Городецкий, Ю. С. Ильяшенко, Некоторые новые грубые свойства инвариантных множеств и аттракторов динамических систем, Функц. анализ и его прил., 33:2 (1999), 16-30.

[15] А. С. Городецкий, Ю. С. Ильяшенко, Некоторые свойства косых произведений над подковой и соленоидом, Труды МИРАН, 231 (2000), 96-118.

[16] А. С. Городецкий, Минималъные аттракторы и частично гиперболические инвариантные множества динамических систем, Дисс. к.ф.-м.н., МГУ им. М. В. Ломоносова, мех.-мат. ф-т, 2001.

[17] А. С. Городецкий, Регулярность иентральных слоев частично гиперболических множеств и приложения, Изв. РАН, сер. матем., 70:6 (2006), 19-44.

[18] А. Городецкий, Ю. Ильяшенко, В. Клепцын, М. Нальский, Неустранимость нулевых показателей Ляпунова, Функц. анализ и его прил., 39:1 (2005), 27-38.

[19] Yu. Ilyashenko, W. Li, Nonlocal bifurcations, Amer. Math. Soc., Providence, R.I., 1998.

[20] А. Б. Каток, Б. Хасселблат, Введение в современную теорию динамических систем, Факториал, М., 1999.

[21] А. Б. Каток, А. М. Степин, Об аппроксимациях эргодических динамических систем периодическими преобразованиями, ДАН СССР, 171 (1966), 1268-1271.

[22] А. Б. Каток, А. М. Степин, Аппроксимации в эргодической теории, УМН, 22(137):5 (1967), 81-106.

Université de Genève

Московский государственный университет

Поступило в редакцию

Независимый московский университет

UMPA ENS Lyon (UMR 5669 CNRS)

e-mail: kleptsyn@mccme.ru

Московский государственный университет

Независимый московский университет

e-mail: maxim@mccme.ru 